\title{
Adherence: the Holy Grail?
}

\section{Christine M Bond}

\section{Correspondence to}

Professor Christine M Bond,

Centre for Academic Primary Care, University of Aberdeen, Aberdeen, UK; c.m.bond@abdn. ac.uk

Accepted 15 February 2016 Published Online First

3 March 2016

\section{SLinked}

- http://dx.doi.org/10.1136/ bmjqs-2015-004400

- http://dx.doi.org/10.1136/ bmjqs-2015-004670

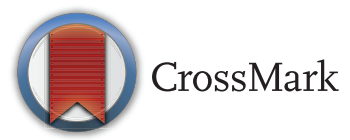

To cite: Bond CM. BMJ Qual Saf 2016;25:736-738.
Adherence to medicines has been identified as a key issue by the Royal Pharmaceutical Society of Great Britain, ${ }^{1}$ the $\mathrm{WHO}^{2}$ and, most recently, by the UK Academy of Medical Sciences. ${ }^{3}$ Estimates of adherence vary widely but are often reported to be as low as $50 \% .{ }^{4}$ When a patient does not take their prescribed medicines as intended, they may not derive the expected outcomes. As well as implications for the individual patient, this can result in increased costs associated with patient hospitalisations or avoidable escalation in other costs of care. ${ }^{3}$ It may also result in unused medicines, the cost of which is estimated at $£ 300$ million per year in England alone. ${ }^{3}$

Causes of non-adherence are multifactorial. Patients have a right to take or not take medicines and may have logical reasons for their behaviour, for example, fear of, or avoidance of, side effects. Many patients do not take opioid analgesics as frequently as prescribed because of worries about addiction; others may not take a diuretic in the morning if they are going out all day, others may not take a medicine because they perceive it to be ineffective. Non-adherence due to such reasons is known as intentional nonadherence. Other causes of nonadherence are unintentional such as forgetting, challenges in swallowing or simply just having difficulties taking a medicine out of a blister pack. However, whether the reasons for non-adherence are intentional or unintentional, there is a responsibility on the part of the healthcare team to empower the patient to discuss these and, in partnership with the patient, come to an agreed plan to address the patient's goals.

A Cochrane review ${ }^{5}$ of interventions to enhance adherence suggests there is no single solution, and only a minority of identified studies improved both adherence and clinical outcomes. Interventions included both face to face and virtual media. Successful interventions were reportedly complex and unlikely to be implementable in routine clinical practice. They need to be tailored to the patient, theory driven and repeated for sustained effect. Pharmacists have been identified as one provider who can support improved adherence with some evidence of benefit.

Adherence is very hard to measure, and there is no gold standard method. The least intrusive method uses prescribing or dispensing records to establish a medication possession ratio, ${ }^{6}$ but a limitation of this method is that it quantifies the numbers of tablets requested or collected without understanding whether or when these have actually been taken by the patient. Another light touch outcome is self-report, and some self-report measures have been validated against physiological readings such as blood pressure. ${ }^{7}$ However, self-report measures are likely to overestimate adherence due to social desirability bias and generally do not consider the timing of doses. With respect to confirming exactly when tablets are taken from their containers, digital bottle tops or blister packs are currently the best method, but even these do not provide any information on whether the tablet has actually been ingested. This can only be reliably confirmed with a blood test or other biomarker, and this may still give misleading results because different individuals metabolise drugs differently. Because none of these measures is perfect, it is generally recommended that two contrasting measures of adherence are used.

For research to change policy and practice, it has to be relevant to a policy need, disseminated to the appropriate stakeholders and have a strong and clearly presented evidence base. Pragmatically, policymakers often want results quickly and cannot always wait for a full randomised controlled trial to be undertaken, following the guidance of the Medical Research Council framework ${ }^{8}$ and including development work and pilot studies prior to the full trial. Ideally, study outcomes should be patient centred, demonstrating an effect on quality of life or a 
clinical outcome. The measures used should also be in line with those recommended for use in formal health technology assessments, for example, those undertaken for the National Institute for Health and Care Excellence (NICE) in the UK or the Canadian Agency for Drugs and Technology in Health. Historically, health services research undertaken in the pharmacy setting has been criticised for dependence on using process measures such as numbers of problems identified or medicines stopped rather than more patientcentred outcomes.

Research can interact or inform policy and practice at several different time points on the pipeline to service redesign. ${ }^{9}$ First, 'blue sky' research can identify new approaches to delivering care and can prompt policy change. A good example of this would be the role of community pharmacists in smoking cessation. ${ }^{10}$ Second, research can be commissioned to support an existing policy idea, recognising that that policy idea might have come from an earlier researcher-led 'blue sky' study. Third, research can be used to evaluate a new area of practice introduced to address an identified policy need but without the underpinning research, such as non-medical prescribing in the $\mathrm{UK}^{11}$; in such an instance, the research may have the additional objective of informing refinement of the policy concerned.

With respect to adherence to medication, one randomised controlled trial in particular has been influential in developing a formal role for community pharmacists in England. ${ }^{12}$ In this study, patients over 75 years, receiving a first prescription for a newly prescribed medicine for a long-term condition, were telephoned after 2 weeks by a pharmacist from a centralised telephone service to discuss their medication-related needs. When patients were followed up by a researcher after 4 weeks, there was significantly less non-adherence in the intervention group compared with the control group. Building on this study, the English New Medicines Service ${ }^{13}$ was introduced, with community pharmacists paid an additional professional fee for its delivery. In the New Medicines Service, eligible patients-those receiving a prescription for a new (to them) medicine for one of the target conditions of asthma/chronic obstructive pulmonary disease, diabetes (type 2), antiplatelet/anticoagulant therapy or hypertension-receive two follow-up consultations from their local pharmacist (either face to face or by telephone) to discuss adherence and other medicine-related issues. Thus, the introduced service was similar to, but not exactly the same, as the one reported in the earlier randomised controlled study. ${ }^{12}$

Against this background, two studies of pharmacy-led adherence interventions are reported in this issue. The evaluation of the English New Medicines Service ${ }^{14}$ is an example of commissioned research evaluating an implemented policy, based on earlier research ${ }^{12}$ as described above. The study design was pragmatic and is an interesting example of how to conduct a randomised controlled trial in the context of a service already universally implemented. Unusually, the unit of randomisation was the patient; normally for a similar research question in an intervention-naive population, a clustered randomisation would have been preferable, removing the potential for contamination within a pharmacy. In this instance, however, this would have had the challenge of finding a way of compensating pharmacists who were financially disadvantaged by being in a control group and not delivering the new service. Randomisation by patient rather than by pharmacy has the potential to reduce the effect size due to intra-cluster (in this case, the community pharmacy) contamination. Since the trial reported here showed quite a large effect, then in this instance there was either little contamination or the true effect was larger than that reported. Other strengths of the research include the use of two different adherence measures (although both are based on self-report), delivery of the intervention by a large number of practising community pharmacists and a health economic assessment. However, limitations include the lack of a pilot study, which is generally acknowledged to be an essential precursor to a full study ${ }^{8}$ to ensure the research processes (eg, recruitment approaches, randomisation and follow-up) work in practice. Furthermore, it did not include any clinical outcomes although it did report patient quality of life using the NICE-recommended measure of the EuroQol. ${ }^{15}$ The primary outcome of self-reported adherence was improved in the group receiving the pharmacist intervention, although there was no change in patient quality of life or in patients' beliefs about medicines, which could have provided an explanation for the observed effect on adherence.

The second study ${ }^{16}$ took the learning from the original study by Clifford $e t$ al ${ }^{12}$ and used a centralised telephone follow-up but applied the intervention to patients already established on a long-term medicine (either a lipid-lowering medicine or an antidiabetic medicine), who had been dispensed a repeat prescription from a mail order pharmacy. In other words, it was an adherence-focused medication review for a patient with a long-term condition and stable therapy. It was strong in design because it used two contrasting measures of adherence, was geographically diverse and had an internal pilot. Conversely, limitations included delivery of the intervention by one highly trained pharmacist and no health economic assessment. Again the study findings show improved adherence by self-report measures, but less strong improvement based on the medication possession ratio and only a nonstatistically significant suggestion of benefit from blood tests. 
So what should we conclude from these two studies? There are clear indications from both studies that pharmacy-based interventions can reduce nonadherence, and from one ${ }^{14}$ that this is a cost-effective approach. However, absolute quantification of benefit is still not possible because of limitations in both studies, and as the authors of the paper on the evaluation of the New Medicines Service ${ }^{14}$ speculate, there are other changes that could be introduced that might lead to further benefits. As the authors suggest, general practitioners should routinely direct patients prescribed a new medicine to receive the service and this could be operationalised by electronic access to a patient's record from the community pharmacy. The second study provided further evidence of the value of an adherence-focused medication review and for follow-up of those on repeat, as opposed to new, medicines, as is already undertaken by community pharmacies in both the UK ${ }^{17} 18$ and elsewhere. It will also inform policy for mail order pharmacies. Both studies are valuable additions to the evidence base for clinical roles for community pharmacists. While the studies were conducted in England, there are also important messages for policymakers worldwide seeking a solution to the global problem of nonadherence to medicines.

Competing interests None declared.

Provenance and peer review Commissioned; internally peer reviewed.

\section{REFERENCES}

1 Royal Pharmaceutical Society of Great Britain. From compliance to concordance: towards shared goals in medicine taking. London: RPS, 1997.

2 World Health Organisation. Adherence to long-term therapies: evidence for action. 2003. http://www.who.int/chp/knowledge/ publications/adherence_introduction.pdf ?ua $=1$ (accessed 20 Jan 2016).

3 Academy of Medical Sciences. Patient adherence to medicines Summary of a joint meeting. 2014. http://www.acmedsci.ac.uk/ viewFile/552f6b3fdab3a.pdf (accessed 20 Jan 2016).

4 Naderi SH, Bestwick JP, Wald DS. Adherence to drugs that prevent cardiovascular disease: meta-analysis on 376,162 patients. Am J Med 2012;125:882-7.e1.
5 Nieuwlaat R, et al. Interventions for enhancing medication adherence. The Cochrane Collaboration, 2014. Published by John Wiley \& Sons, Ltd (Review). http://onlinelibrary.wiley. com/doi/10.1002/14651858.CD000011.pub4/epdf (accessed 20 Jan 2016).

6 Andrade SE, Kahler KH, Frech F. Methods for evaluation of medication adherence and persistence using automated databases. Pharmacoepidemiol Drug Saf 2006;15:565-74; discussion 575-7.

7 Morisky DE, Ang A, Krousel-Wood M, et al. Predictive validity of a medication adherence measure for hypertension control. J Clin Hypertens 2008;10:348-54.

8 Craig P, Dieppe P, Macintyre S, et al. Developing and evaluating complex interventions: the new Medical Research Council guidance. BMJ 2008;337:a1655.

9 Bond CM. Pharmacy practice research: evidence and impact. In: Babar Z, ed. Pharmacy Practice Research Methods Switzerland: Springer International Publishing, 2015.

10 Sinclair HK, Bond CM, Stead LF. Community pharmacy personnel interventions for smoking cessation (Review). The Cochrane Collaboration. Issue 2, 2008.

11 Latter S, Blenkinsopp A, Smith A, et al. Evaluation of nurse and pharmacist independent prescribing. http://eprints.soton. ac.uk/184777/2/ENPIPexecsummary.pdf (accessed 4 Feb 2016).

12 Clifford S, Barber N, Elliott R, et al. Patient centred advice is effective in adhering adherence to medicines. Pharm World Sci 2006;28:165-70 (accessed 4 Feb 2016).

13 New Medicines Service. http://psnc.org.uk/services-commissioning/ advanced-services/nms/

14 Elliot RA, Boyd MJ, Saleme NE, et al. Supporting adherence for people starting a new medication for a long term condition through community pharmacies: a pragmatic randomised controlled trial of the New Medicines Service. BMJ Qual Saf 2016;25:747-58.

15 Euroqol. http://www.euroqol.org/ (accessed 4 Feb 2016).

16 Lyons I, Barber N, Raynor DK, et al. The Medicines Advice Service Evaluation (MASE): a randomised controlled trial of a pharmacist led telephone based intervention designed to improve medication adherence. BMJ Qual Saf 2016;25:759-69.

17 Medication Use Review. http://psnc.org.uk/servicescommissioning/advanced-services/murs/ (accessed 21 Jan 2016).

18 Chronic Medication Service. http://www.communitypharmacy scotland.org.uk/nhs-care-services/services/chronic-medicationservice/what-is-the-chronic-medication-service/ (accessed 4 Feb 2016). 